\title{
COMPORTAMIENTO ELECTORAL DE LOS COLOMBIANOS DURANTE LAS ELECCIONES PRESIDENCIALES DEL AÑO 2010*
}

DOI: http://dx.doi.org/10.17981/juridcuc.11.1.2015.9

Recibido: 26 de Mayo de 2015 / Revisado: 07 de Julio de 2015 / Aceptado: 09 de Julio de 2015

\section{Roberto Ochoa Villa** \\ Universidad de Zulia}

Puede citar el presente artículo así: / To reference this article:

Ochoa, R. (2015). Comportamiento Electoral de los Colombianos durante las elecciones presidenciales del año 2010. Jurídicas CUC, 11(1), 209-220. doi: http://dx.doi.org/10.17981/juridcuc.11.1.2015.9

\section{Resumen}

El presente artículo pretende desentrañar cómo incidieron los factores sociales y demográficos o sociológicos en la decisión del voto de los colombianos durante la elección presidencial del 2010. El análisis se hace sobre los resultados de una encuesta de intención de voto realizada por la firma Gallup Colombia, a partir de los cuales se concluye que la crisis total de la nación que se expresa en la crisis de los partidos políticos, ha venido generando un estado de descontento continuo o endémico como consecuencia de la insatisfacción de las necesidades colectivas y de la cual se hacen agentes los partidos políticos, ante la situación social de los habitantes o de los electores, todo esto ha afectado la institucionalidad de tal forma que, al desaparecer las diferencias entre los partidos tradicionales, el descontento se expresa en la alta abstención para votar por los partidos hegemónicos.

\section{Palabras claves:}

Participación electoral, descontento endémico, crisis, sistema de partidos, fragmentación, volatilidad, partidos hegemónicos.

\footnotetext{
*Artículo de reflexión realizado con recursos propios del autor.

** Doctorante en Ciencia Política en la Universidad del Zulia, Especialista en Planificación Territorial en Universidad del Magdalena, especialista en métodos y técnicas avanzadas de Investigación en Ciencias Sociales y la Educación. Presidente de la Asociación de Sociología del Caribe ASOCIOCARIBE; Docente Catedrático en la Facultad de Derecho Universidad de la Costa - CUC Barranquilla. Docente Catedrático en las Facultades de Educación e Ingeniería de la Universidad de La Guajira. Correo electrónico: rochoavilla@hotmail.com
} 


\section{ELECTORAL BEHAVIOR OF COLOMBIANS DURING THE PRESIDENTIAL ELECTIONS IN 2010}

\section{Abstract}

This article aims to unravel as influenced social and demographic or sociological in decision Colombians vote during the presidential election of 2010 factors. Analyses were done on the results of a poll of likely voters conducted by the Gallup Colombia, from which we conclude that the total crisis of the nation that is expressed in the crisis of political parties, has been generating a state of continuous or endemic discontent as a result of dissatisfaction of collective needs and which agents make political parties, to the social situation of the citizens or voters, this has affected the institutions so that the disappearance of the differences between the traditional parties expressed dissatisfaction in high abstention to vote for mainstream parties.

\section{Keywords:}

Voting endemic unrest, crisis, party system fragmentation, volatility, mainstream parties. 


\section{INTRODUCCIÓN}

Tratar de abordar el comportamiento político electoral de una población, desde la perspectiva de su análisis como hecho político, está encaminado en definitiva en la vía de, ya sea "adivinar" o predecir el comportamiento futuro o de pretender el análisis de un hecho electoral ya sucedido en cualquier espacio o lugar de nuestro país.

Desde luego que pretender explicar analizando el comportamiento electoral de los votantes en las elecciones de 2010 acaecidas en la República de Colombia para elegir presidente, hace necesario la explicación de la arqueología crítica de los partidos políticos existentes en el país, su relación con los electores, el papel cumplido en la historia y transformaciones de la sociedad colombiana y el efecto electoral en la motivación para participar en el evento de los votantes.

Es así, como los partidos liberal y conservador han cumplido un papel fundamental en la construcción de la historia política del país, lo cual los ha llevado a un desgaste ante la sociedad colombiana en las últimas cinco décadas, pero es claro que este fenómeno político no es exclusivo de la realidad política colombiana, sino que parece ser frecuente e intenso en los países subdesarrollados de América Latina y el Caribe (Molina, 1997) como consecuencia del ejercicio continuo del poder político, ya sea alternadamente o en posiciones de bipartidismo.

La insatisfacción de las necesidades de la población y el incumplimiento de las expectativas de los electores hace posible la aparición de un malestar y rechazo de los partidos políticos por parte de los electores, modificando su posición hegemónica de poder, lo cual es resultado de lo que algunos autores denominan "descontento endémico" (Molina, 2000); es así como observando las cifras de Latinbarómetro $2002^{1}$, en Colombia, el porcentaje de confianza en los partidos políticos pasó de $29,08 \%$ en 1997 a 10,01\% en 2002, lo cual nos muestra una variación significativa en el porcentaje, producto de la crisis y el desgaste de los partidos tradicionales en Colombia.

\footnotetext{
${ }^{1}$ Latinbarómetro es un estudio de opinión pública política que se aplica anualmente a cerca de 19.000 entrevistas en 18 países de A.L., representa a más de 400 millones de habitantes.
} 
No se puede desconocer que la situación sociopolítica en el país en los últimos 50 años se ha presentado en un marco de violencia política y partidista, tampoco debemos ignorar que aunque la génesis de los partidos políticos tradicionales en Colombia, Liberal y Conservador, vienen del siglo XIX, ellos no han podido darle respuesta a las demandas y expectativas de los electores y ciudadanos en general. Esta democracia, que parece ser una de las más antiguas en Latinoamérica, está en la región del mundo que tiene la característica de presentar las mayores desigualdades económicas, políticas y sociales.

De acuerdo con la información en la Tabla 1, podemos encontrar cómo la participación de los ciudadanos en los eventos electorales en Colombia cada día disminuye, incrementando la abstención.

El sistema tradicional de partidos políticos en Colombia, que ha estado constituido por los partidos Liberal y Conservador, logró desarrollar una gran identificación partidaria en una considerable porción de la población de votantes hasta los últimos 30 años, presentándose institucionalizados, constituidos legalizados y ligeramente organizados para acceder al poder de manera continua o alternadamente, ya sea a través de elecciones democráticas donde ellos se presentan en la competencia o a través de lo que desde los años 50 del siglo XX llamaron Frente Nacional, en el que se cerraron las posibilidades de participación de partidos diferentes a ellos o al bipartidismo, lo cual hizo que su diferencia programática desapareciera.

Los partidos políticos Conservador y Liberal que se presentaban como unas organizaciones de masas con alguna estructura organizacional fuerte y estable en las que primaban los elementos ideológicos, hoy se presentan como organizaciones de cuadros con estructuras precarias, poco disciplinados, carentes de contenidos programáticos que logran permanecer en el escenario político al consolidar algún liderazgo de una persona de gran influencia en la circunscripción electoral (Duvergier, 1988). Este parece ser el nuevo escenario de los partidos políticos colombianos, pues ante el desgaste surge una gran fragmentación que origina una volatilidad muy dinámica del voto. 
Tabla 1.

Participación electoral en América Latina 1978 y 2006.

\begin{tabular}{|c|c|c|c|c|}
\hline \multirow[b]{2}{*}{ País } & \multicolumn{2}{|c|}{ Elecciones presidenciales } & \multicolumn{2}{|c|}{ Elecciones legislativas } \\
\hline & $\begin{array}{c}\text { Año } \\
\text { electoral }\end{array}$ & $\begin{array}{c}\% \text { participación } \\
\text { de votantes }\end{array}$ & $\begin{array}{c}\text { Año } \\
\text { electoral }\end{array}$ & $\begin{array}{c}\text { \% participación } \\
\text { de votantes }\end{array}$ \\
\hline Colombia & $\begin{array}{l}1978,1982, \\
1986,1990, \\
1994,1998, \\
2002,2006\end{array}$ & 44,5 & $\begin{array}{c}1978,1982, \\
1986,1990, \\
1991,1994, \\
1998,2002, \\
2006\end{array}$ & 42,8 \\
\hline Promedio América Latina & & 72,95 & & 2,95 \\
\hline
\end{tabular}

Fuente: Daniel Zovatto. Participación electoral en América Latina: tendencias y perspectivas 1978-2002; y Daniel Zovatto. Región: crece el abstencionismo electoral.

La crisis del bipartidismo ve respuesta en una salida de modernización del sistema de partidos en Colombia, lo cual trae como consecuencia una apertura política que permite ampliar la participación de nuevas opciones partidistas, hecho que fue afianzado por la Constitución de 1991, propiciando así una pluralidad en el aumento de nuevas fuerzas políticas como expresión de movimientos sociales, coaliciones, personalismos, entre otros. Esta nueva situación aún no logra resolver el nivel de abstención presentado en los diferentes eventos electorales de tipo partidista y al distribuirse la participación de los electores en las diferentes opciones en competencia, desde luego que existe un alto grado de inestabilidad en la competición partidista, los resultados ahora son menos predecibles y es más fácil para los nuevos partidos su entrada a la escena electoral y la competición por el poder (Mainwaring, citado por Hoyos, 2005). En consecuencia, se hace necesario plantearse el siguiente interrogante: ¿cuáles son los factores que incidieron en los colombianos al momento de tomar sus decisiones de voto en las elecciones de 2010 ?

\section{Enfoques sobre el comportamiento electoral}

El comportamiento electoral ha sido uno de los fenómenos políticos más estudiados de la Ciencia Política. Los primeros análisis datan desde los inicios del siglo XX y desde entonces la Ciencia Política ha ido desarrollando el marco teórico y metodológico para explicar el comportamiento 
electoral. Según Salamanca (2012), inicialmente los análisis consistían en la recopilación de datos electorales que permitían establecer la geografía electoral ${ }^{2}$ de los comicios, luego, con los estudios de la Universidad de Columbia se avanzó hacia la sociología electoral. Posteriormente, con el desarrollo de las técnicas de encuestas y de los sondeos de opinión pública, se dio un impulso definitivo de la Ciencia Política al permitir la adquisición de información desde la perspectiva individual (Dalton \& Wattemberg, 1993).

Una búsqueda de los estudios sobre el comportamiento electoral permite determinar la existencia, inicialmente, de tres grandes enfoques teóricos que buscan explicar los factores que están detrás de la racionalidad de las personas al momento de tomar sus decisiones de voto: el enfoque sociológico, el enfoque psicológico y el enfoque económico/racional, además del enfoque institucional. Sus fundamentos son expuestos a continuación:

El enfoque sociológico: Escuela de Columbia. Este enfoque teórico fue desarrollado por Paul Lazarsfeld y Bemald Berelson, profesores de la Universidad de Columbia en Nueva York, quienes en su obra, El pueblo elige, plantearon un esquema analítico sobre las elecciones presidenciales norteamericanas que enfatizaba el rol y la influencia de los grupos primarios y las características sociales de los ciudadanos en sus decisiones electorales.

El enfoque psicológico: Escuela de Michigan. Este enfoque hace énfasis en la identificación partidista -el enfoque de Campbel y asociados- y constituye una explicación de la conducta electoral a partir del eslabonamiento de factores descritos en términos de un embudo causal que tiene un eje temporal (hechos históricos de largo y corto plazo) sobre el cual se van agregando una serie de factores.

La explicación se mueve entre las características del sistema político, el perfil sociodemográfico del elector, el contexto histórico y los factores psicológicos, privilegiando estos últimos (Salamanca, 2012)

\footnotetext{
${ }^{2}$ Esta tendencia ha sido renovada en la actualidad con la incorporación de novedosas técnicas computarizadas que amplían sus posibilidades de aplicación y análisis. (Pérez, 2006)
} 
El enfoque de la decisión racional: económico. La teoría económica se ha construido sobre el supuesto de prevalencia de la racionalidad consciente. Si el teórico conoce los objetivos del sujeto de decisión, puede prever qué acciones emprenderá para lograrlo, y para eso, primero calcula la trayectoria más razonable para llegar a tales objetivos, y segundo, supone que seguirá esa trayectoria por tratarse de un sujeto racional (Downs, 1957). De esta manera, las decisiones de voto son el resultado de un análisis de los beneficios que obtiene y los costos que incluye tal decisión.

El enfoque institucional. Este enfoque, desde luego, muestra cómo las instituciones pueden motivar o restringir la intensión o decisión al voto de los electores y el comportamiento electoral en general. Ya sea, formas de gobierno, sistemas electorales o sistemas de partidos. No podemos desconocer que las instituciones son pautas de conducta reiteradas, estables y apreciadas, de igual manera, ellas son el proceso por el que adquieren valor y estabilidad las organizaciones y procedimientos (Huntington, 1975).

\section{Aspectos Metodológicos y Operativos}

Para realizar el presente análisis se parte de información recogida en una encuesta aplicada por la firma Gallup en el mes de marzo de 2010 en las principales ciudades de Colombia, la cual tiene un margen de error del $0.05 \%$ y un nivel de confianza del $95.5 \%$. El muestreo se hizo de manera probabilística a una muestra escogida, también se basa en información reportada por la agencia estatal de la Registraduria Nacional.

\section{Análisis de los resultados}

Al analizar el contenido de la Tabla 2 acerca de la probabilidad de voto de los colombianos según su posición en la estratificación social, lo cual según Anduiza \& Bosh (2004) pueden condicionar su voto, se encuentra que existe una tendencia alta positiva a votar en las elecciones presidenciales. En la muestra se observa una probabilidad de voto mucho más significativa en los estratos bajo y medio bajo, mientras que para los estratos medio y medio alto la probabilidad es muy baja. 
Tabla 2.

Relación de la probabilidad de votar de los colombianos por estrato social

\begin{tabular}{cccccccc}
\hline $\begin{array}{c}\text { Probabilidad de } \\
\text { votar }\end{array}$ & $\begin{array}{c}\text { Bajo- } \\
\text { bajo }\end{array}$ & $\begin{array}{c}\text { Bajo- } \\
\text { medio }\end{array}$ & $\begin{array}{c}\text { Medio- } \\
\text { bajo }\end{array}$ & Medio & $\begin{array}{c}\text { Medio- } \\
\text { alto }\end{array}$ & Alto & Total \\
\hline Sí votaría & $75.0 \%$ & $72.8 \%$ & $74.8 \%$ & $76.5 \%$ & $83.8 \%$ & $100.0 \%$ & $75.0 \%$ \\
\hline No votaría & $10.2 \%$ & $17.6 \%$ & $16.9 \%$ & $18.9 \%$ & $10.8 \%$ & $.0 \%$ & $15.9 \%$ \\
\hline Indeciso & $13.1 \%$ & $9.1 \%$ & $8.3 \%$ & $3.8 \%$ & $2.7 \%$ & $.0 \%$ & $8.5 \%$ \\
\hline NS/NR & $1.7 \%$ & $.4 \%$ & $.0 \%$ & $.8 \%$ & $2.7 \%$ & $.0 \%$ & $.6 \%$ \\
\hline Total encuestado & 176 & 449 & 385 & 132 & 37 & 21 & 1200 \\
\hline $\begin{array}{c}\text { Probabilidad de } \\
\text { votar }\end{array}$ & $14.7 \%$ & $37.4 \%$ & $32.1 \%$ & $11.0 \%$ & $3.1 \%$ & $1.8 \%$ & $100 . \%$ \\
\hline
\end{tabular}

Fuente: Estudio de Gallup de marzo de 2010.

De igual manera, se muestra que según el género no se observa diferencia en la probabilidad de votar. Desde luego, este factor que es de largo plazo como lo es el género (Pérez, 2008), algunos autores afirman que lo femenino presenta baja participación electoral, lo simpático en esta encuesta es que en ella se iguala la participación electoral de género.

Tabla 3.

Relación de la intención de voto de los colombianos por género.

\begin{tabular}{cccc}
\hline Probabilidad de votar & Femenino & Masculino & Total \\
\hline Sí votaría & $71.8 \%$ & $78.2 \%$ & $75.0 \%$ \\
\hline No votaría & $16.5 \%$ & $15.3 \%$ & $15.9 \%$ \\
\hline Indeciso & $10.8 \%$ & $6.2 \%$ & $8.5 \%$ \\
\hline NS/NR & $.8 \%$ & $.3 \%$ & $.6 \%$ \\
\hline Total encuestado & 600 & 600 & 1200 \\
\hline Probabilidad de votar & $50.0 \%$ & $50.0 \%$ & $100.0 \%$ \\
\hline
\end{tabular}

Fuente: Estudio de Gallup de marzo de 2010.

De otra parte, no se encuentra diferencia significativa en la intención positiva de voto para la elección a Presidente por los diferentes grupos de edades, claro que a pesar de la cercanía entre cifras por los diferentes grupos etarios en su intención de voto, encontramos que los grupos de edades entre 25-35 hasta 44 años y los de 55 años y más, presentan una tendencia de probabilidad de votar alta, mientras ésta disminuye para los otros dos grupos de edades; su identificación subjetiva hace que varíe entre grupos (Pérez, 2008). 
Tabla 4.

Relación de la intención de voto de los colombianos por grupo edad en las elecciones presidenciales del 2010.

\begin{tabular}{ccccccc}
\hline Probabilidad de votar & $\mathbf{1 8 - 2 4}$ & $\mathbf{2 5 - 3 4}$ & $\mathbf{3 5 - 4 4}$ & $\mathbf{4 5 - 5 4}$ & $\mathbf{5 5}$ o más & Total \\
\hline Sí votaría & $71.0 \%$ & $75.2 \%$ & $73.5 \%$ & $73.5 \%$ & $75.6 \%$ & $75.0 \%$ \\
\hline No votaría & $18.2 \%$ & $15.8 \%$ & $17.7 \%$ & $10.6 \%$ & $16.4 \%$ & $15.9 \%$ \\
\hline Indeciso & $10.3 \%$ & $8.6 \%$ & $8.5 \%$ & $8.6 \%$ & $6.9 \%$ & $8.5 \%$ \\
\hline NS/NR & $.5 \%$ & $.4 \%$ & $.4 \%$ & $.5 \%$ & $1.1 \%$ & $.6 \%$ \\
\hline Total encuestado & 214 & 266 & 260 & 198 & 262 & 1200 \\
\hline Probabilidad de votar & $17.8 \%$ & $22.2 \%$ & $21.7 \%$ & $16.5 \%$ & $21.8 \%$ & $100.0 \%$ \\
\hline
\end{tabular}

Fuente: Estudio de Gallup de marzo de 2010.

Teniendo en cuenta la actividad u ocupación de los encuestados, se observa diferencia significativa en su opción y probabilidad de voto para las elecciones presidenciales. Al comparar, amas de casa y trabajadores señalan tendencias positivas, mientras que los desempleados, estudiantes y pensionados presentan poco interés probable de votar; es posible que no estuviesen lo suficientemente motivados por las opciones de voto.

Tabla 5.

Relación de la intención de voto de los colombianos por ocupación o actividad.

\begin{tabular}{ccccccc}
\hline $\begin{array}{c}\text { Probabilidad } \\
\text { de votar }\end{array}$ & $\begin{array}{c}\text { Ama de } \\
\text { casa }\end{array}$ & Desempleado & Estudio & Pensionado & Trabajo & Total \\
\hline Sí votaría & $69.4 \%$ & $75.5 \%$ & $72.5 \%$ & $80.3 \%$ & $77.4 \%$ & $75.0 \%$ \\
\hline No votaría & $17.3 \%$ & $13.6 \%$ & $15.8 \%$ & $15.8 \%$ & $15.7 \%$ & $15.9 \%$ \\
\hline Indeciso & $11.6 \%$ & $10.9 \%$ & $10.8 \%$ & $3.9 \%$ & $6.7 \%$ & $8.5 \%$ \\
\hline NS/NR & $1.8 \%$ & $.0 \%$ & $.8 \%$ & $.0 \%$ & $.2 \%$ & $.6 \%$ \\
\hline Total encuestado & 284 & 110 & 120 & 76 & 610 & 1200 \\
\hline $\begin{array}{c}\text { Probabilidad de } \\
\text { votar }\end{array}$ & $23.7 \%$ & $9.2 \%$ & $10.0 \%$ & $6.3 \%$ & $50.8 \%$ & $100.0 \%$ \\
\hline
\end{tabular}

Fuente: Estudio de Gallup de marzo de 2010.

De igual forma, según el grado de educación o formación existe diferencia en la opción de voto para las elecciones presidenciales, presentándose los dos primeros niveles de educación con la mayor intención de voto para las elecciones de 2010. Este hecho podría comprenderse desde la perspectiva que significa estrenarse en la actividad de votar, parece haber un cálculo racional utilitario en el hecho electoral (Downs, 1973) o en la intención. 
Tabla 6.

Relación de la intención de voto de los colombianos por nivel educativo.

\begin{tabular}{cccccc}
\hline $\begin{array}{c}\text { Probabilidad de } \\
\text { votar }\end{array}$ & $\begin{array}{c}\text { Primaria o } \\
\text { menos }\end{array}$ & Secundaria & $\begin{array}{c}\text { Técnico/ } \\
\text { tecnológico }\end{array}$ & Profesional & Total \\
\hline Sí votaría & $69.7 \%$ & $70.0 \%$ & $80.4 \%$ & $87.2 \%$ & $75.0 \%$ \\
\hline No votaría & $18.3 \%$ & $19.3 \%$ & $11.6 \%$ & $9.4 \%$ & $15.9 \%$ \\
\hline Indeciso & $0.8 \%$ & $10.1 \%$ & $8.0 \%$ & $3.0 \%$ & $8.5 \%$ \\
\hline NS/NR & $1.2 \%$ & $.6 \%$ & $.0 \%$ & $.4 \%$ & $.6 \%$ \\
\hline Total encuestado & 251 & 513 & 199 & 234 & 1200 \\
\hline Probabilidad de votar & $20.9 \%$ & $42.8 \%$ & $16.6 \%$ & $19.5 \%$ & $100.0 \%$ \\
\hline
\end{tabular}

Fuente: Estudio de Gallup de marzo de 2010.

Asimismo, al observar los resultados de la participación de la población en las elecciones presidenciales de manera comparativa entre los años 2006 y 2010, podemos implicar que la abstención en ambos años, a pesar de que en 2010 se presentan dos vueltas (en el 2006 triunfa Álvaro Uribe en la primera vuelta), se observa que el nivel de la participación en las elecciones se mantiene en el mismo porcentaje, al igual que la abstención.

Tabla 7.

Información general de la elección presidencial 2006 - 2010.

\begin{tabular}{ccccccc}
\hline Variables & $\mathbf{2 0 0 6}$ & $\mathbf{\%}$ & $\begin{array}{c}\text { Primera } \\
\text { vuelta 2010 }\end{array}$ & $\mathbf{\%}$ & $\begin{array}{c}\text { Segunda } \\
\text { vuelta 2010 }\end{array}$ & $\%$ \\
\hline Potencial electoral & 26.731 .700 & & 29.983 .279 & & 29.983 .279 & \\
\hline Total votos & 12.041 .737 & 100 & 13.337 .658 & & 13.337 .658 & \\
\hline Votos candidatos & 11.638 .113 & 97 & 12.593 .040 & 96 & 12.593 .040 & 96.58 \\
\hline Votos blancos & 226.297 & 2 & 445.330 & 3.41 & 445.330 & 341 \\
\hline Votos nulos & 132.332 & 1 & 199.302 & 1.49 & 199.302 & 1.49 \\
\hline Tarjetones no marcados & 44.995 & 0 & 99.998 & 0.74 & 99.986 & 0.74 \\
\hline Participación electoral & & 45 & & 49 & & 44.4 \\
\hline Abstención & & 55 & & 51 & & 55.6 \\
\hline
\end{tabular}

Fuente: Registraduria Nacional del Estado Civil.

Con el análisis de las dos vueltas de las elecciones de 2010, se encuentra que el candidato más opcionado y con mayor votación en ambos eventos representa la continuidad de los gobiernos de 2002 y 2006 , 
pues Juan Manuel Santos se desempeñó como Ministro de Defensa del gobierno de Uribe, mientras que Antanas Mockus presenta una opción un poco alejada del proyecto continuista. Aunque la opción de voto por el candidato del gobierno es altamente elegida, superando al otro candidato casi tres veces, la abstención se sigue presentando en una alta proporción, razón por la cual, ésta parece ser la verdadera ganadora. El efecto de persuasión por las opciones de los electores (Anduiza \& Bosch, 2004) no los mueve, lo cual mantiene la abstención en iguales parámetros históricos.

Tabla 8.

Resultados en las dos vueltas de las elecciones de 2010.

\begin{tabular}{ccccc}
\hline Candidato & 2010 Primera vuelta & \% & 2010 Segunda vuelta & \% \\
\hline Juan Manuel Santos & 6.802 .043 & 47 & 9.004 .221 & $69,05 \%$ \\
\hline Antanas Mockus & 3.143 .222 & 22 & 3.588 .819 & $27,52 \%$ \\
\hline
\end{tabular}

Fuente: Registraduria Nacional del Estado Civil.

Por último, encontramos que la participación de los electores se vuelca en un $63 \%$ entre los partidos de la U, Liberal y Conservador, obteniendo estos 63 escaños para Senado en 2010, lo cual es superior a los 56 logrados en 2006; el resto de opciones partidarias recibe el saldo de votos minoritarios depositados en las urnas en ambas elecciones. Esto ilustra la dispersión y volatilidad del voto en las últimas elecciones en Colombia.

\section{CONCLUSIONES}

Durante las elecciones realizadas en 2010 en Colombia para escoger al Presidente de la República, se pudo observar que la crisis total de la nación que se expresa en la crisis de los partidos políticos, ha venido generando un estado de descontento continuo o endémico como consecuencia de la insatisfacción de las necesidades colectivas y ante la situación social de los habitantes o de los electores, de la cual se hacen agentes los partidos políticos. Esto ha afectado la institucionalidad de tal forma que, al desaparecer las diferencias entre los partidos tradicionales, el descontento se expresa en la alta abstención para votar por los partidos hegemónicos, además, al presentarse procesos de apertura política o 
de modernización del sistema electoral en Colombia, la desconfianza o la pérdida de creencia de los electores hace que se presente una alta fragmentación en las opciones políticas de éstos, y como consecuencia, la volatilidad es un fenómeno corriente que hoy se expresa en las opciones personalistas o coyunturales que se le presentan a los votantes, lo cual parece que es la punta del iceberg de la situación política nacional.

\section{REFERENCIAS}

Anduiza, E. \& Bosch, A. (2004). Comportamiento Político y Electoral. Barcelona: Editorial Ariel S.A.

Dalton, R. \& Wattemberg, M. (1993). The Not So Simple Act Of Voting. Political Science: The State of The Discipline. 2 ed. Washington D.C: The American Political Sciencie Association.

Downs, A. (1957). An Economic Theory of Democracy. New York: Haper Collins.

Downs, A. (1973). Teoría Económica de la Democracia. Madrid: Ed. Aguilar.

Duvergier, M. (1988). Instituciones Políticas y Derecho Constitucional. Barcelona: Ariel.

Hoyos, D. (2005). Evolución del Sistema de Partidos en Colombia, 1972-2000. Una mirada Local y Regional. Revista Análisis Políticos, 55. Bogotá: Universidad Nacional de Colombia.

Huntington, S. (1975). The United States. The crisis of Democracy. New York: Trilateral Commission.

Molina, J. (1977). El Efecto del Subdesarrollo y sus Causas en América Latina, El Caribe y los Países industrializados, Cuaderno de Cendes, 36. Caracas: Universidad Central de Venezuela.

Pérez, C. (2006). Enfoques teóricos-metodológicos en el estudio de la participación electoral. Cuestiones Políticas, 37, 74-93. Maracaibo: Universidad del Zulia.

Pérez, C. (2008). Curso sobre campañas electorales y análisis del comportamiento electoral. Maracaibo: Universidad Católica Cecilio Acosta.

Salamanca, L. (2012). ¿Por qué vota la gente?. Caracas: El Alfa. 